\title{
In vitro comparison of antibacterial properties of bioceramic-based sealer, resin-based sealer and zinc oxide eugenol based sealer and two mineral trioxide aggregates
}

\author{
Gurpreet Singh ${ }^{1}$, Iti Gupta ${ }^{1}$, Faheim M. M. Elshamy ${ }^{2}$, Nezar Boreak ${ }^{1}$, \\ Husham Elraih Homeida ${ }^{3}$
}

Correspondence: Dr. Gurpreet Singh Email: drgurpreetsingh@gmail.com

\begin{abstract}
'Department of Endodontics, College of Dentistry, Jazan University, Jizan, Kingdom of Saudi Arabia, ${ }^{2}$ Department of Dental Materials, College of Dentistry, Jazan University, Jizan, Kingdom of Saudi Arabia, ${ }^{3}$ Department of Microbiology, College of Dentistry, Jazan University, Jizan, Kingdom of Saudi Arabia
\end{abstract}

\section{ABSTRACT}

Objective: Our goal of the study was to evaluate the antibacterial properties of endodontic sealers against the E. faecalis. Materials and Methods: Six millimeters wells were made for each material in all the preinoculated petri plates. Then, the petri plates were incubated for $24 \mathrm{~h}$. The zones of inhibition appeared were measured, and the measurements were put to statistical analysis. Results: EndoSequence BC Sealer, MM-mineral trioxide aggregate (MTA), and ProRoot MTA showed maximum means of diameter of zones of inhibition, whereas MM-seal and Endoseal did not show any zones of inhibition. Conclusion: EndoSequence $\mathrm{BC}$ Sealer was found to be a better endodontic sealer as compared to resin-based and zinc oxide-eugenol-based sealer.

Key words: Antibacterial, endodontic sealer, EndoSequence BC Sealer, Enterococcus faecalis, mineral trioxide aggregate

\section{INTRODUCTION}

The successful endodontic treatment in infected teeth depends on the elimination of the microbial load by the chemomechanical preparation of the root canals. However, the complete elimination of microorganism from the root canal system is not possible in all the cases. Endodontic infections are polymicrobial, and more than 150 species of bacteria and other microorganisms are present that are responsible for the primary or persistent infection. ${ }^{[1]}$ Enterococcus faecalis is a commonly isolated species that may play a

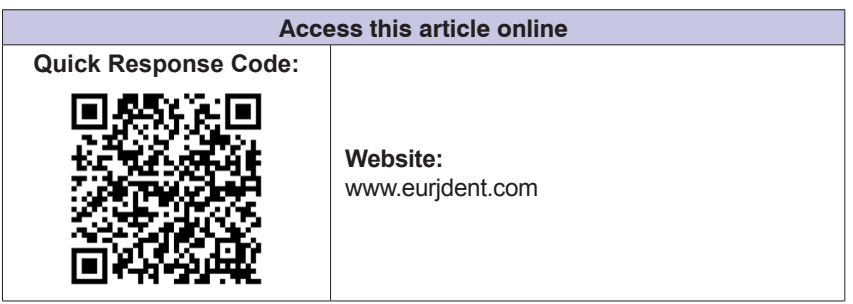

role in persistent endodontic infections. Studies report a prevalence of $E$. faecalis up to $77 \%$ in teeth with failed endodontic treatment. ${ }^{[2,3]}$

The use of root canal filling materials having antimicrobial activity is considered advantageous in the effort to reduce the number of remaining microorganisms, prevent recurrent root canal infection, and aid in the healing of periapical tissues. ${ }^{[4]}$

This is an open access article distributed under the terms of the Creative Commons Attribution-NonCommercial-ShareAlike 3.0 License, which allows others to remix, tweak, and build upon the work non-commercially, as long as the author is credited and the new creations are licensed under the identical terms.

For reprints contact: reprints@medknow.com

How to cite this article: Singh G, Gupta I, Elshamy FM, Boreak N, Homeida HE. In vitro comparison of antibacterial properties of bioceramicbased sealer, resin-based sealer and zinc oxide eugenol based sealer and two mineral trioxide aggregates. Eur J Dent 2016;10:366-9.

DOI: $10.4103 / 1305-7456.184145$ 
EndoSequence BC Sealer (Brasseler, Savannah, GA, USA) is a premixed bioceramic endodontic sealer that is mainly composed of zirconium oxide, calcium silicates, calcium phosphate monobasic, calcium hydroxide, filler, and thickening agents. ${ }^{[5]}$ Bioceramic sealer has now become popular in endodontics as root repair material and root canal sealer due to its several advantages such as biocompatibility, high $\mathrm{pH}(<12)$, nonshrinkable, nonresorbable, ease of delivery in the root canal, and increase in strength of the root the following obturation. ${ }^{[6,7]}$

Endoseal (Prevest Denpro, Jammu, India) is a zinc oxide-eugenol-based permanent root canal sealar with dexamethasone, thymol iodide, hydrocortisone acetate, bismuth subcarbonate, epoxy resins, barium sulfate, and magnesium stearate. According to the manufacturer, it is antibacterial, anti-inflammatory sealant with extended working time, and long setting time.

MM-SEAL (Micro Mega, France) is an epoxy resin-based, paste/ paste sealer for permanent filling of root canals using Gutta-percha. According to the manufacturer, it is eugenol-free, biocompatible and radio-opaque.

Mineral trioxide aggregate (MTA) has been proved to exhibit excellent biocompatibility and capacity for inducing mineralized tissue formation and has been used for sealing perforations, for root-end filling, and in apexification cases. ${ }^{[8]}$ MTA has demonstrated favorable clinical outcomes in early researches; therefore, other uses for MTA were explored and investigated. MTA has been used as root canal obturation material ${ }^{[9]}$ and as root canal sealer after modifications in the original formulation to improve its characteristics. ${ }^{[10]}$ It was demonstrated that MTA was very useful as an obturation material in previously treated teeth that are compromised by microleakage, inadequate cleaning and shaping, poor quality obturations, and large periapical lesions. ${ }^{[9]}$

Many studies have been performed to assess the antibacterial activity of different endodontic sealers by different methods. There is little information available about the comparison of the antibacterial properties of the materials used in this study. The goal of the study was to compare the antibacterial activity of the newly introduced EndoSequence BC Sealer (Brasseler, Savannah, GA, USA), MM sealer (Micro Mega, France), Endoseal (prevest denpro, Jammu, India), ProRoot white MTA (Dentsply, Ballaigues,
Switzerland), and MM-MTA (Micro Mega, France) against $E$. faecalis.

\section{MATERIALS AND METHODS}

\section{Materials used}

1. EndoSequence BC sealer (Brasseler, Savannah, GA, USA)

2. MM seal (Micro mega, France)

3. Endoseal (Prevest Denpro, Jammu, India)

4. MM-MTA (Micro Mega, France)

5. ProRoot white MTA (Dentsply, Ballaigues, Switzerland)

6. Microorganism used:

a. E. faecalis (ATCC 29212).

The activity of the materials was evaluated by the method of agar diffusion test. A base layer of $10 \mathrm{ml}$ of Mueller-Hinton agar was poured in $10 \mathrm{~mm} \times 100 \mathrm{~mm}$ sterilized petri plates. The second layer or seed layer containing $10 \mathrm{ml}$ of Mueller-Hinton agar was poured after the solidification of agar and the microbial standardized suspensions were poured. Then, five wells of $6 \mathrm{~mm}$ of diameter (one for each material) were obtained by removing the agar at equidistant points with the help of sterilized plastic straw. The wells were immediately filled with all the five materials to be evaluated. The endodontic materials Endoseal, MM seal, and two types of MTA that are MM-MTA and ProRoot MTA were prepared according to the manufacturer's instruction, whereas EndoSequence BC Sealer is a premixed paste.

The plates were incubated at $37^{\circ} \mathrm{C}$ for $24 \mathrm{~h}$. The inhibition zones around the well were measured with millimeter ruler. The data were collected and subjected to the statistical analysis. Mean (X) and standard deviations were calculated. Chi-square test was done to evaluate intraobserver bias for all the study samples. Intergroup comparison was done using Pearson correlation statistical analysis. All statistical analyses were performed with the SPSS 20.0 statistical software package (IBM). $P<0.05$ was considered statistically significant.

\section{RESULTS}

The mean zone of the inhibition recorded from the study has been illustrated in Table 1 .

The results of our study showed that the maximum inhibitory activity was shown by the EndoSequence BC Sealer $(14.4 \mathrm{~mm})$ followed by MM-MTA $(14.3 \mathrm{~mm})$ 


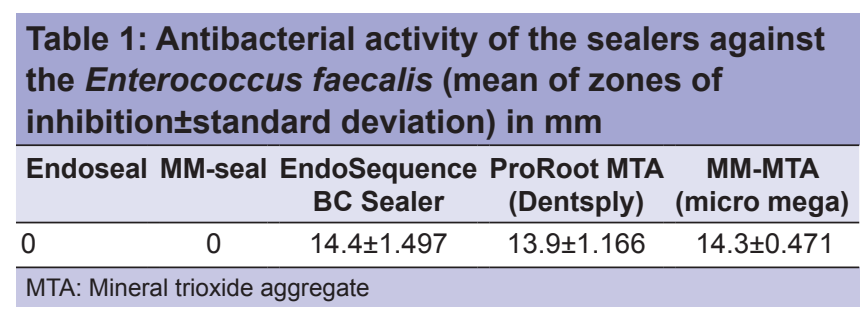

and the ProRoot MTA the value is $13.9 \mathrm{~mm}$. However, there was no statistically significant difference between these three materials $(P>0.05)$. However, the results showed that no inhibitory activity was found with Endoseal $(0 \mathrm{~mm})$ and $\mathrm{MM}$ - seal $(0 \mathrm{~mm})$ against the $E$. faecalis. The statistical analysis could not be done with these two groups.

\section{DISCUSSION}

E. faecalis is a Gram-positive nonspore forming facultative anaerobe bacterium. It has an ability to survive harsh environments. It can resist bile salts, detergents, heavy metals, ethanol, azide \& desiccation. ${ }^{[11]}$ E. faecalis is also resistant against calcium hydroxide, a commonly used intracanal medicament because of its proton pump which maintains the $\mathrm{pH}$ level. ${ }^{[12]}$ It forms the biofilm which protects it from the host defense and antimicrobial agents. ${ }^{[13]}$ It penetrates deep into the dentinal tubules and invades isthmuses, rami, and accessory canals. Many studies have confirmed its presence in failed root canal treatment cases. ${ }^{[2,14]}$

The agar diffusion method was used in our study as it is one of the most commonly used techniques for evaluating the antimicrobial properties of the material. However, this method does not provide an opportunity for the consideration of factors such as anatomy of the tooth and biofilm formation by the microorganism. ${ }^{[15]}$

Our study showed that the maximum mean size of inhibition zone was found with the EndoSequence BC sealer $(14.4 \mathrm{~mm})$ followed by MM-MTA $(14.3 \mathrm{~mm})$ and ProRoot MTA (13.9 mm). But the results were not significant statistically. This is attributed to high $\mathrm{pH}$ released by these materials that promote the elimination of bacteria like $E$. faecalis that do not survive high $\mathrm{pH}$ near 11.5 or more. ${ }^{[16]}$ Lovato and Sedgley compared antibacterial property of bioceramic root repair material and MTA and their result illustrated similar inhibitory effect against the E. faecalis. ${ }^{[17]}$ These results are in accordance with our study. Many recent studies have demonstrated similar antibacterial effect against the E. faecalis between bioceramic material and MTA. ${ }^{[18,19]}$
MTA was introduced as root perforation material by Lee et al. in 1993. ${ }^{[20]}$ Further, it gained popularity as pulp capping agent, for pulpotomy, apexification, and as root-end filling material because of its bioactivity and biocompatibility. Now, MTA is being used as the obturation material ${ }^{[9]}$ as well as root canal sealer. ${ }^{[21]}$ Holland et al. demonstrated in their study that MTA used as root canal sealer has potential to regenerate the periodontal ligament and form cementum in the canal system which closes the empty spaces thus, prevent the treatment failure. ${ }^{[22]}$ Yildirim and Gencoglu showed successful use of MTA as apical filling material in cases of large apical lesion after 6 years of follow-up. ${ }^{[23]}$ Duarte et al. demonstrated the mechanism of increase in $\mathrm{pH}$ and formation of calcium and hydroxide ions in MTA, which is responsible for the antibacterial effect of MTA. They explained that calcium oxide when reacts with water forms calcium hydroxide which induces high $\mathrm{pH}$ that results its dissociation into calcium and hydroxide ions. ${ }^{[24]}$

Epoxy resin-based sealers have antimicrobial effects related to either bisphenol A diglycidyl ether or the release of formaldehyde during polymerization. In our study, MM-seal (Micro mega) did not show any inhibitory effects against E. faecalis. Leonardo et al. studied the amount of release of the formaldehyde of the four different endodontic sealers and found that epoxy resin-based sealer showed minimum amount of release of formaldehyde during setting. ${ }^{[25]}$ This may be the reason that epoxy resin-based sealer used in this study showed no antibacterial activity against the E. faecalis. Pizzo et al. reported that epoxy resin-based sealer had no inhibitory effect against $E$. faecalis after $24 \mathrm{~h},{ }^{[26]}$ which is consistent with our current findings. Slutzky-Goldberg et al. tested four endodontic sealers against $E$. faecalis and found that epoxy resin-based sealer $\mathrm{AH}$ plus showed no antibacterial activity. ${ }^{[27]}$ Zhang et al. compared the antibacterial effectiveness of the seven different endodontic sealers using the direct contact method at different time intervals and their results showed that after $24 \mathrm{~h}$, bioceramic-based sealer showed statistically significant high antibacterial activity, whereas epoxy resins-based sealer failed to show any inhibitory effect on the E. faecalis. ${ }^{[4]}$ Ustun et al. evaluated the antibacterial effectiveness of four different sealers AH Plus, EndoRez, MTA Fillapex, iRoot SP against $E$. faecalis by time-kill assay method in vitro. They showed that at $20 \mathrm{~min}$, the MTA-based sealer was ineffective, whereas bioceramic sealer and epoxy resin sealer were bactericidal. However, after 7 days and 30 days, MTA-based sealer was bactericidal but all other sealers bacteriostatic. ${ }^{[28]}$ 
Endoseal (Prevest Denpro), a zinc oxide-eugenol-based sealer did not show any inhibitory effect on the E. faecalis in our study. The size of the zone of inhibition depends on two main factors that are toxicity of the material to a particular strain of the bacteria and the ability of the material to diffuse through the particular medium. The diffusibility in turn is affected by three main factors that are hydrophobicity or hydrophilicity of the material, size, and rate of release from the matrix in which the material is put. These factors are difficult to control when the test is being done in vitro. Considering these factors, this test may show different results with different sealers. ${ }^{[29]}$ Wang et al. evaluated the antibacterial effect of four endodontic root canal sealers on E. faecalis biofilm in dentinal tubules and found that zinc oxide-eugenol sealer has weaker antibacterial effect as compared to other sealers. They explained the reason on the basis of sensitivity of the technique used to test materials. ${ }^{[15]}$ Similarly, Tabrizizadeh and Mohammadi demonstrated low antibacterial effect of zinc oxide-eugenol-based sealer in their study. ${ }^{[30]}$ It should be noted that the size of the inhibition zones does not show the exact antimicrobial effect of a sealer. The root canal sealers evaluated in this study; therefore, may show different inhibitory effects against $E$. faecalis in vivo.

\section{CONCLUSION}

The bioceramic root canal sealer has similar antibacterial activity as that of both MTAs but better than other two most common types of sealers used. Further research is required to compare their efficacy in vivo.

\section{Financial support and sponsorship}

Nil.

\section{Conflicts of interest}

There are no conflicts of interest.

\section{REFERENCES}

1. Mattigatti S, Jain D, Ratnakar P, Moturi S. Antimicrobial effect of conventional root canal medicament vs. propolis against Enterococcus faecalis, staph aureus and candida albicans. J Contemp Dent Pract 2012;13:305-9.

2. Hancock HH, Sigurdsson A, Trope M, Moiseiwitsch J. Bacteria isolated after unsuccessful endodontic treatment in a North Am population. Oral Surg Oral Med Oral Pathol Oral Radiol Endod 2001;91:579-86.

3. Stuart CH, Schwartz SA, Beeson TJ, Owatz CB. Enterococcus faecalis: Its role in root canal treatment failure and current concepts in retreatment. J Endod 2006;32:93-8.

4. Zhang H, Shen Y, Ruse ND, Haapasalo M. Antibacterial activity of endodontic sealers by modified direct contact test against Enterococcus faecalis. J Endod 2009;35:1051-5.

5. Loushine BA, Bryan TE, Looney SW, Gillen BM, Loushine RJ,
Weller RN, et al. Setting properties and cytotoxicity evaluation of a premixed bioceramic root canal sealer. J Endod 2011;37:673-7.

6. Nasseh AA. The rise of bioceramics. Endod Prac 2009;8;21-5.

7. Candeiro GT, Correia FC, Duarte MA, Ribeiro-Siqueira DC, Gavini G. Evaluation of radiopacity, $\mathrm{pH}$, release of calcium ions, and flow of a bioceramic root canal sealer. J Endod 2012;38:842-5.

8. Torabinejad M, Watson TF, Pitt Ford TR. Sealing ability of a mineral trioxide aggregate when used as root end filling material. J Endod 1993;19:591-5.

9. Bogen G, Kuttler S. Mineral trioxide aggregate obturation: A review and case series. J Endod 2009;35:777-90.

10. Zhou HM, Du TF, Shen Y, Wang ZJ, Zheng YF, Haapasalo M. In vitro cytotoxicity of calcium silicate-containing endodontic sealers. J Endod 2015;41:56-61.

11. Hegde V. Enterococcus faecalis: Clinical significance and treatment considerations. Endodontology 2009;21:48-52.

12. Evans M, Davies JK, Sundqvist G, Figdor D. Mechanisms involved in the resistance of Enterococcus faecalis to calcium hydroxide. Int Endod J 2002;35:221-8.

13. George S, Kishen A, Song KP. The role of environmental changes on monospecies biofilm formation on root canal wall by Enterococcus faecalis. J Endod 2005;31:867-72.

14. Sundqvist G, Figdor D, Persson S, Sjögren U. Microbiologic analysis of teeth with failed endodontic treatment and the outcome of conservative re-treatment. Oral Surg Oral Med Oral Pathol Oral Radiol Endod 1998;85:86-93.

15. Wang Z, Shen $Y$, Haapasalo M. Dentin extends the antibacterial effect of endodontic sealers against Enterococcus faecalis biofilms. J Endod 2014;40:505-8.

16. McHugh CP, Zhang P, Michalek S, Eleazer PD. pH required to kill Enterococcus faecalis in vitro. J Endod 2004;30:218-9.

17. Lovato KF, Sedgley CM. Antibacterial activity of endosequence root repair material and proroot MTA against clinical isolates of Enterococcus faecalis. J Endod 2011;37:1542-6.

18. Charland T, Hartwell GR, Hirschberg C, Patel R. An evaluation of setting time of mineral trioxide aggregate and EndoSequence root repair material in the presence of human blood and minimal essential media. J Endod 2013;39:1071-2.

19. Willershausen I, Wolf T, Kasaj A, Weyer V, Willershausen B, Marroquin BB. Influence of a bioceramic root end material and mineral trioxide aggregates on fibroblasts and osteoblasts. Arch Oral Biol 2013;58:1232-7.

20. Lee SJ, Monsef M, Torabinejad M. Sealing ability of a mineral trioxide aggregate for repair of lateral root perforations. J Endod 1993;19:541-4

21. Gomes-Filho JE, Watanabe S, Bernabé PF, de Moraes Costa MT. A mineral trioxide aggregate sealer stimulated mineralization. J Endod 2009;35:256-60.

22. Holland R, de Souza V, Nery MJ, Otoboni Filho JA, Bernabé PF, Dezan Júnior E. Reaction of dogs' teeth to root canal filling with mineral trioxide aggregate or a glass ionomer sealer. J Endod 1999;25:728-30.

23. Yildirim T, Gencoglu N. Use of mineral trioxide aggregate in the treatment of large periapical lesions: Reports of three cases. Eur J Dent 2010;4:468-74.

24. Duarte MA, Demarchi AC, Yamashita JC, Kuga MC, Fraga Sde C. pH and calcium ion release of 2 root-end filling materials. Oral Surg Oral Med Oral Pathol Oral Radiol Endod 2003;95:345-7.

25. Leonardo MR, Bezerra da Silva LA, Filho MT, Santana da Silva R. Release of formaldehyde by 4 endodontic sealers. Oral Surg Oral Med Oral Pathol Oral Radiol Endod 1999;88:221-5.

26. Pizzo G, Giammanco GM, Cumbo E, Nicolosi G, Gallina G. In vitro antibacterial activity of endodontic sealers. J Dent 2006;34:35-40.

27. Slutzky-Goldberg I, Slutzky H, Solomonov M, Moshonov J, Weiss EI, Matalon S. Antibacterial properties of four endodontic sealers. J Endod 2008;34:735-8.

28. Ustun Y, Sagsen B, Durmaz S, Percin D. In vitro antimicrobial efficiency of different root canal sealers against Enterecoccus faecalis. European J Gen Dent 2013;2:134-8.

29. al-Khatib ZZ, Baum RH, Morse DR, Yesilsoy C, Bhambhani S, Furst ML. The antimicrobial effect of various endodontic sealers. Oral Surg Oral Med Oral Pathol. 1990;70:784-90.

30. Tabrizizadeh M, Mohammadi Z. In vitro evaluation of antibacterial activities of root canal sealers. J Clin Dent 2005;16:114-6. 